\title{
ACCRETION FLOWS IN MAGNETIC CATACLYSMIC VARIABLES
}

\author{
A. J. Norton, ${ }^{1}$ R. V. Somerscaless. ${ }^{1}$ T. L. Parker, ${ }^{1}$ G. A. Wymn. ${ }^{2}$ and R. G. West ${ }^{2}$
}

\section{RESUMEN}

Presentamos los resultarlos dr simulaciones que exploran la variedad de flujos de acreción posibles en variables caliclísmicas malgnétim (mCVs). Nuestras simulaciones evolucionan hasta alcanzar períodos de equilibrio que abarcan $0.01<P_{\text {spin }} / P_{\text {orb }}<0.6$, y los flujos resultantes varían desde sistemas alimentados por disco en $P_{\text {spin }} / P_{\text {orb }} \sim 0.01-0.1$ a sistemas alimentados por corrientes en $P_{\text {spin }} / P_{\text {orb }} \sim 0.1-0.5$ y sistemas alimentados doside un anillo en el borde cxtorior del lóbulo de Roche de la enana blanca, en $P_{\text {spin }} / P_{\text {orb }} \sim 0.6$.

\section{ABSTRACT}

Wr present the results of simulations that explore the variety of accretion flows possible in magnetic cataclysmic varialles (mCVs). Our simulations evolve to equilibrium periods spanning $0.01<P_{\text {spin }} / P_{\text {orb }}<0.6$ and the resulting flows vary from disc:-fed systems at. $P_{\text {spin }} / P_{\text {orb }} \sim 0.01-0.1$, to stream fed systems at $P_{\text {spin }} / P_{\text {orb }} \sim$ $0.1-0.5$ and systems ferd from a ring at the outer edge of the white dwarf's Roche lobe at $P_{\text {spin }} / P_{\text {orb }} \sim 0.6$.

kiy Words: ACCRETION, ACCRETION DISCS

\section{INT'RODUTCTION}

IPs are semi-cletached interacting binaries in which a magnetic: white dwarf (WD) aceretes material from a late-type, low mass, secomelary star via Roche lobe overflow. They are distinguished from the other sub-class of $111 \mathrm{C} \mathrm{V}$ s. the polars, in that the spin periods of the WISs in IPs are loss than their binary orbital periods (in polars, $P_{\mathrm{sp} \text { in }}=P_{\text {orb }}$ ) and they have somewhat lower WD magnetic field strengths. In all m( $V$ 's, when material reaches the magnetospheric radius of the WD, it couples to the field lines and thereafter is channelled onto the magnetic pole(s) of the Wil) where it undergoes a strong sloock before sectling on the surface and cooling by the cmission of X-lay bremsstrahlung and optical/infrared cyclotron emission. The defining characteristic of IPs is strong X-ray emission pulsed at the white dwate spin period due to phase-dependent visibility of, and absorption cowards, the X-ray emitting regions. Sce Wirner (1995) for a comprehensive review of ('Vis and Patterson (1994) for a summary of the (then known) IPs.

Around 40 IP's alle currently recognised, and one way of characterising them is by their spin to orbital period ratio $\left(P_{\text {spin }} / P_{\text {orl }}\right)$ as shown in Figure 1. As may be seen, all $m$ ('Vis (i.e. IP's and polars) seem to ignore the ustal (' 1 period gap betwe'n 2 and 3 hours. extending across all orbital periods from $\sim 80$ minutes to $\sim 10$ hours. Furthermore. IPs span a range of $P_{\text {spin }} / P_{\text {orb }}$ values from $\sim 0.001-0.9$.

\footnotetext{
${ }^{1}$ Dept. of Physics \& Astronomy: The Open Iniversity:

${ }^{2}$ Dept. of Physics \& Astronomin. Leicester Lniversity:
}

BINARIES: CLOSE - STARS: MAGNETIC

\section{SPIN EQUILIBRIA}

The spin rate of a magnetic WD accreting via a disc reaches equilibrium when the rate of angular momentum accreted by the WD equals the braking effect of the magnetic torque on the disc close to its inner edge. The inner edge of the disc is the point where the magnetic stresses exceed the viscous stresses. Most models find that accreting WDs reach an equilibrium spin period when the inner radius of the disc is approximately equal to the co-rotation radius. This implies that systems with a low WD magnetic field strength have short spin periods, whilst systems with a high WD magnetic field strength have long spin periods. This in turn leads to the requirement for a truncated accretion disc to form of $R_{\text {in }} \sim R_{\text {co }}<<R_{\text {circ }}$ which implies

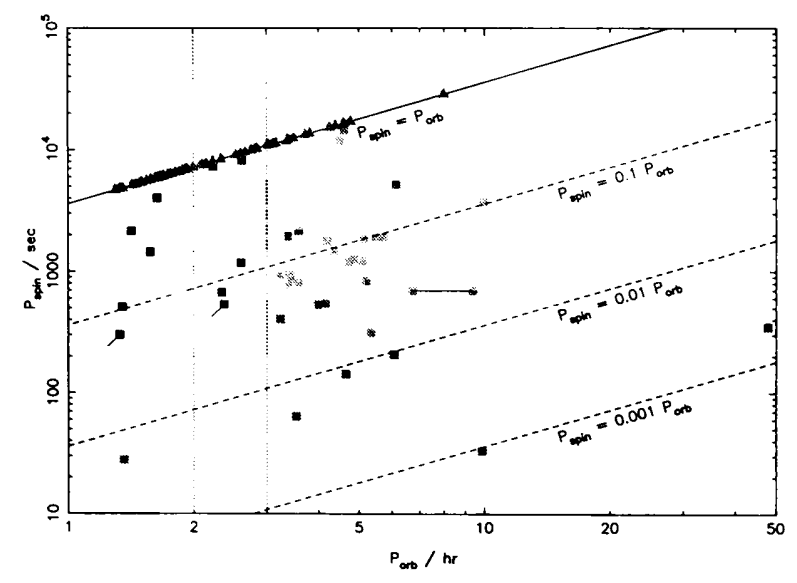

Fig. 1. The spin and orbital periods of all mCVs. Polars are indicated by triangles; IPs by squares. 
$P_{\text {spin }} / P_{\text {orb }}<<0.1$. Whilst this is true for some systems, such as DQ Her, V533 Her and GK Per, it is certainly not true for all IPs (see Figure 1).

Systems with $P_{\text {spin }} / P_{\text {orb }}>0.1$ are unlikely to possess discs, since this implies $R_{\text {in }} \sim R_{\text {co }}>R_{\text {circ }}$. King \& Wynn (1999) showed that EX Hya can be understood as an equilibrium situation with the co-rotation radius extending out to the inner Lagrangian point. Such a system naturally has an equilibrium at $P_{\text {spin }} / P_{\text {orb }} \sim 0.65$, close to that observed.

\section{THE MAGNETIC MODEL}

The equation of motion of plasma in a magnetic field depends on the gas pressure, the magnetic pressure, the magnetic tension and gravitational force. If we assume that the magnetic tension dominates over the magnetic pressure, the magnetic part of the acceleration may be parameterised in terms of a drag parameter, $k$, which, for diamagnetic blobs, is inversely proportional to the magnetic timescale. $k$ may then be expressed in terms of the WD magnetic moment, aild the density and length scale of the blobs of accreting material.

We have used a particle code that incorporates this parameterisation to simulate the accretion flows in mCVs. Runs were evolved to spin equilibrium for a range of ten values of orbital period each with $k$ ranging between $10^{2}$ and $10^{7}$. The resulting equilibria are shown in Figure 2, where the $k$ parameters have been translated into WD magnetic moments by making appropriate assumptions about the blob density and size. In addition, Figure 2 shows the locations of the known IPs which have been added by tracing across at their measured $P_{\text {spin }} / P_{\text {orb }}$ value to intercept the line corresponding to the appropriate $P_{\text {orb. }}$. Assuming each IP is at its equilibrium spin period, this effcctively determines its WD magnetic moment. For comparison, Figure 2 also shows the locations of those polars for which a measured value of the magnetic field strength is available. A more extensive, but somewhat preliminary, report of our simulations may be found in Norton, Somerscales \& Wynn (2003).

The accretion flows corresponding to four locations along the locus at $P_{\text {orb }}=4$ hours are shown in Figure 3. Similar types of accretion flow may be mapped along each of the other lines of constant orbital period. We see that, as expected, for $P_{\text {spin }} / P_{\text {orb }}<0.1$ the accretion flow may broadly be characterised as disc-fed although at the lowest values some material is also spilled from the outer edge of the disc too. At intermediate spin to orbit period ratios in the range $0.1<P_{\text {spin }} / P_{\text {orb }}<0.5$, the

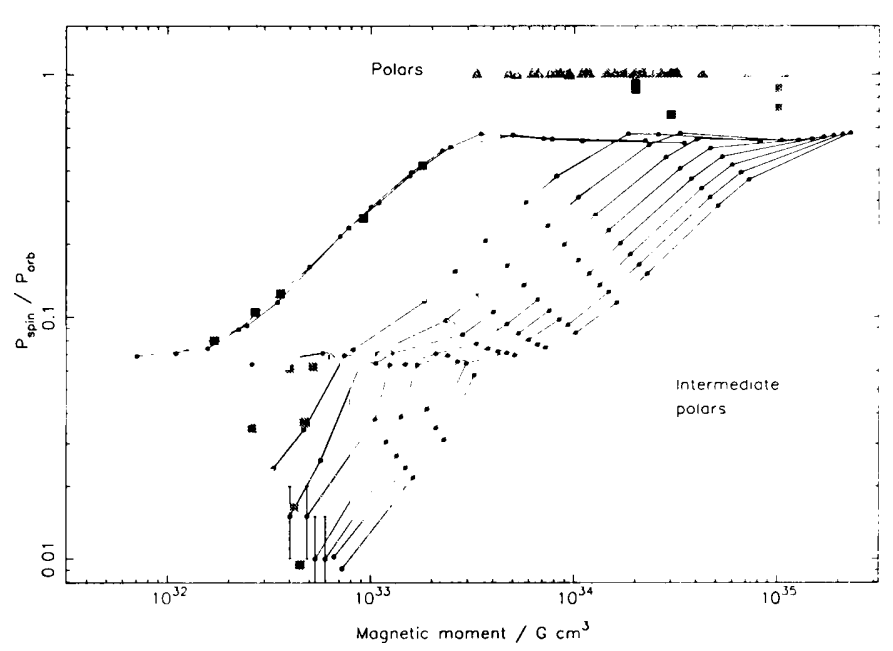

Fig. 2. Results of rumning the magnetic model to find spin cquilibria corresponding to a range of orbital periods and WD magnetic moments. The ten romererel lines correspond to orbital periods of $80 \mathrm{ml}, 2 \mathrm{~h}, 3 \mathrm{~h}, \ldots 10 \mathrm{~h}$, ruming from left to right. The known polat's are shown by triangles and the IPs by squares.
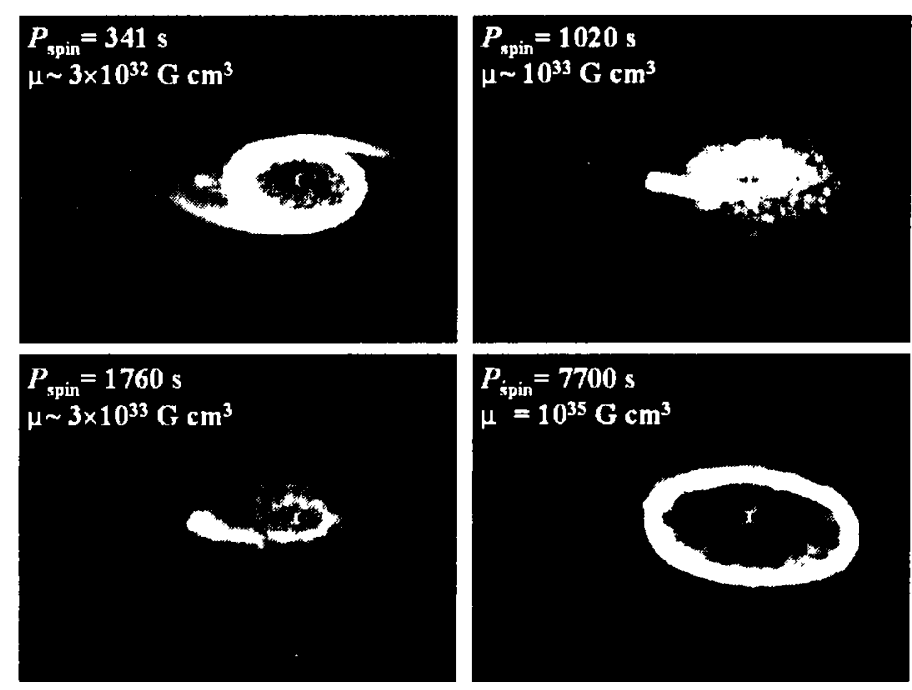

Fig. 3. Example accretion flows at equilibrium for $P_{\text {orb }}=$ $4 \mathrm{hr}$. The equilibrium spin to orbital period ratios are $\sim 0.025,0.07,0.12$ and 0.55 respectively as the WD magnetic moment incrases. Movies of these accretion flows are available at http://physics.open.ac.uk/iphows.html

accretion flow is essentially stream-fed. Finally at $P_{\text {spin }} / P_{\text {orb }} \sim 0.6$, the accorting minterial forms a ring at the outer edge of the WD Roche lobe, from where accretion curtains funnel down to the W1) surface.

\section{REFERENC'ES'}

King, A. R., \& Wynn, G. A. 1999, MNRAS. 310. 203

Norton, A. J.. Somerscales. R.V., \& Wymm, (i. A. 2003. in ASP Conf. Sor. 190, "Vagnetic Cataclysulc Vari-

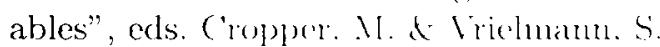

Pirt terson, .J. 1991. PASP, 106, 209

Warner, B. 1995, Cataclysmic lariable Stars. Cambridge Universits Press. Cambridge 